\title{
Evaluation of Proximal Fibular Osteotomy in treatment of Medial Compartment Knee Osteoarthritis
}

\author{
A.H.Adawy, M.I.kandil, S.Abdallah and M.R.Ramzy \\ Orthopedic Surgery Dept., Faculty of Medicine, Benha Univ., Benha, Egypt \\ E-Mail: Mahmoud Rady @gmail.com
}

\begin{abstract}
The administration of older patients with tricompartmental joint inflammation of the knee would preferably be an all out knee arthroplasty. While in more youthful patients with segregated average compartment joint pain of the knee related with a varus deformation, the careful modalities accessible for the executives are restricted to high tibial osteotomy and unicondylar knee substitution. These systems are related with their own arrangement of intricacies just as being related with a more extended postoperative recuperation period and furthermore limitation of exercises or weight bearing, consequently there is a requirement for a technique which is easy to perform, effectively reproducible, gives great useful outcomes and related with a more limited recuperation period and improves the personal satisfaction for the influenced patients. In this situation, (PFO) is a moderately new and novel technique which as indicated by recently distributed and continuous examinations has been demonstrated to be extremely powerful in the administration of average compartment joint pain of the knee Patients and Methods :From January 2018 to june2019, 20 patients who went through proximal fibular osteotomy for average compartment osteoarthritis. Preoperative and postoperative knee society score (KSS) and visual simple scale (VAS) were acquired to survey knee capacities and torment Results: Median knee society score (KSS) show improvement postoperatively from46 to 82 and visual simple scale (VAS) diminished postoperatively from 7 to 1.4. Ends: This investigation shows that proximal fibular osteotomy (PFO), another medical procedure, can adequately diminishes torment and improves knee work in patients with average compartment osteoarthritis.
\end{abstract}

Key words: Early results, Proximal fibular osteotomy, Medial compartment knee O.A.

\section{Introduction}

Proximal fibular osteotomy (PFO) might be an elective treatment to high tibial osteotomy (HTO) [1]. It is a surgery for average compartment knee osteoarthritis (KOA). Contrasted with High Tibial Osteotomy(HTO), PFO has a few focal points [2]. To begin with, the careful strategy is straightforward and effortlessly performed. Second, it is less intrusive with an exceptionally short entry point, requires restricted tissue analyzation and no interior obsession is embedded. The postoperative recuperation period is additionally more limited than with HTO. What's more, the intricacies related with HTO can be a significant issue that adds to a helpless guess [3].

Incontrast, $\mathrm{PFO}$ is related with hardly any entanglements [4] Similar to HTO, PFO can alleviate the side effects of KOA with realignment of the lower limit, while amending the arrangement is the significant goal of HTO [5], the standard of fibular osteotomy starts from the hypothesis of non-uniform settlement [6], Which proposes a critical settlement of the tibial level after osteoporosis. As a result of the help by the fibula, non-uniform implies that the settlement of the level is uneven, with settlement in the average level more clear than in the sidelong plateau.Eventually, the average level turns out to be fundamentally lower than the parallel and a varus deformation happens in the lower limit. These progressions of the mechanical hub lead to a pressure fixation in the average compartment and degeneration of the ligament and meniscus, which are the major obsessive appearances of KOA [6].
Subsequently, in non-uniform-settlement of the tibial level, KOA could likewise be known as a pressure imbalanced condition of the knee joint. PFO debilitates the parallel fibular backing and prompts a rectification of the varus deformation, which can consequently move the stacking power from the average compartment all the more horizontally, prompting diminished torment and a good practical recuperation $\mathrm{PFO}$ is a relatively [1].

Nonetheless, there has been no itemized examination with respect to which patients would accomplish a prevalent forecast after PFO. In this examination, to give a premise to itemized careful sign and patient choice, the relationship between preoperational factors that may influence the forecast of PFO and patients' results is reflectively broke down.

\section{Patients and methods}

20 patients who underwent WITH PFO was admitted to Benha university Hospital and Naser Institude Hospital from January 2018 to June 2019, were included in our study.The patients were all diagnosed according to the criteria of the American college of Rheumatology.

\subsection{The inclusion criteria}

Patients with moderate to severe symptoms of the knee over kellegran and Lawrence (KLgrade)on radiographs (Brandt KD.,1991), age more than 40years, isolated medial compartment arthritis, at least $2 \mathrm{~mm}$ medial joint space present on weight bearing $\mathrm{X}$ rays, BMI less than 32 and varus less than 10" 


\subsection{The exclusion criteria}

Patients with under 40 years, varus more than 10", Bi or Tricompartmental joint pain, dreary corpulence and bone on bone appearance on weight bearing $\mathrm{X}$ rays,rheumatoid arthritis,posttraumatic arthritis,congenital disfigurements of lower limb,joint infection, history of tendon or meniscus injury . On confirmation, all patients were assessed clinically, radiologically and practically. This investigation was endorsed by the moral advisory group of Benha University Hospital and was led as per Declaration Of Helsinki.

\subsection{Clinical assessment}

Clinical information included sexual orientation, age, length of symtoms and evaluating frameworks. The last included Visual simple scale (VAS): which is a self-report measure by quiet himself which went preoperatively from 7 to 10 and following two months postoperatively from 3 to 5 and following four months from 3 to 7. what's more, Knee Society score (KSS score), which comprised of both clinical score and capacity score( Insall JN, 1989). The clinical score of KSS major incorporates agony, solidness and scope of movement. Also, the capacity score centers around the exercises of the patient.

\subsection{Radiographic evaluation}

- plain $\mathrm{x}$ ray (both knee AP 124tan 124tanding position, lat. View,skyline view,Ap in $45^{\circ}$, )

- CT if needed,MRI if needed.

In this study, radiological factors included hipknee-ankle angle (HKA angle),(Hunter DJ.,2005),KL grade(Brandt KD.,1991) ,joint space width of both compartments (Tourville TW.,2013) . The HKA angle was measured on spliced images of total length lower extremities and all other measurements were made on weight-bearing AP radiographs(4) .A goniometer or length meter of the Synapase System (Fujifilm medical systems)was used for measurement. To test the intraand inter-observer reproducibility, two orthopedic surgeons (who are not related to surgery) performed all of the radiographic measurements Briefly, the medial joint space was determined by a vertical line (A) between two horizontal lines $(C$ and $D)$ that were drawn from the lowest point of the medial condyle of the femur and medial plateau of the tibia, respectively. The lateral joint space was determined by a vertical line (B) between two horizontal lines ( $\mathrm{E}$ and $\mathrm{F}$ ) that were drawn from the lowest point of the lateral condyle of the femur and lateral plateau of the tibia, respectively. The ratio of the knee joint space (medial/lateral) was determined by the ratio of $\mathrm{A} / \mathrm{B}$ (Figure 9). Data collection and assessment were performed by two independent observers who were not involved in the surgery.

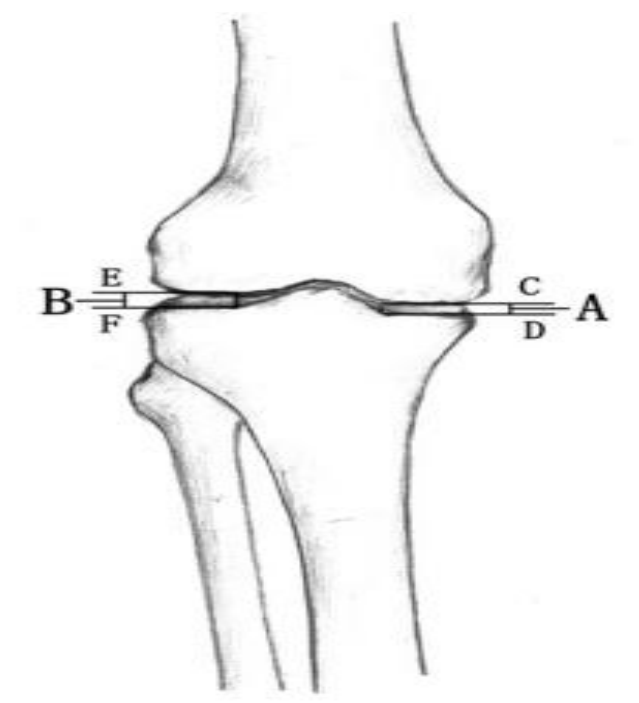

Fig (1) Measurement of ratio of knee joint space. The medial joint space was determined by a vertical line (A) between two horizontal lines $(\mathrm{C}$ and $\mathrm{D})$ that were drawn from the lowest point of the medial condyle of the femur and medial plateau of the tibia, respectively. The lateral joint space was determined by a vertical line (B) between two horizontal lines (E and F) [14].

\subsection{Surgical technique}

After obtaining informed consent and anaesthetic fitness, the patients were taken up for the procedure. The surgery was performed with the patient in the supine position under spinal anaesthesia with antibiotic cover. Tourniquet was not used routinely in our series.
The fibular head was marked and the osteotomy site was determined to be 7 to $9 \mathrm{~cm}$ from the head of fibula. The rationale behind choosing this level of osteotomy is that an osteotomy at a higher level would be likely to cause an injury to the common peroneal nerve while if it was done any lower down that the effect of the 
osteotomy on the medial compartment arthritis would be lost. A 5-8 cm lateral incision was made overlying the chosen site of osteotomy and dissection was carried out through the skin and subcutaneous tissues. The peroneus and soleus muscles were then separated to expose the periosteum of the fibula which was then incised and a 1.5 to $2 \mathrm{~cm}$ of fibula was then resected with the help of an osscilating saw after placing a few drill holes at the osteotomy site. Fig (2) Curved homann retractors were placed behind the fibula prior to osteotomy and care was taken not to stretch the soft tissues too much in order to protect the nerve from potential damage. Occasionally after the osteotomy some of the fibulae tend to bleed quite profusely and in that situation bone wax was used to seal the cut ends of the bone. After ensuring heamostasis and giving wound wash, closure was done in layers and sterile dressing and compression bandage applied.

\subsection{Postoperative care and follow up}

All patients were encouraged to stand and walk on the same evening of surgery and were discharged on the third postoperative day after the first wound inspection. Intravenous antibiotics for given for 3 days followed by oral antibiotics for a period of 5 days. The sutures were removed on the $12^{\text {th }}$ postoperative day. Postoperative weight bearing $X$ rays were then taken and the radiological parameters were evaluated and documented. The patients were reviewed at 1, 3, 6 months and at the end of the first year where the VAS and the knee society scores were evaluated and documented Fig (2).

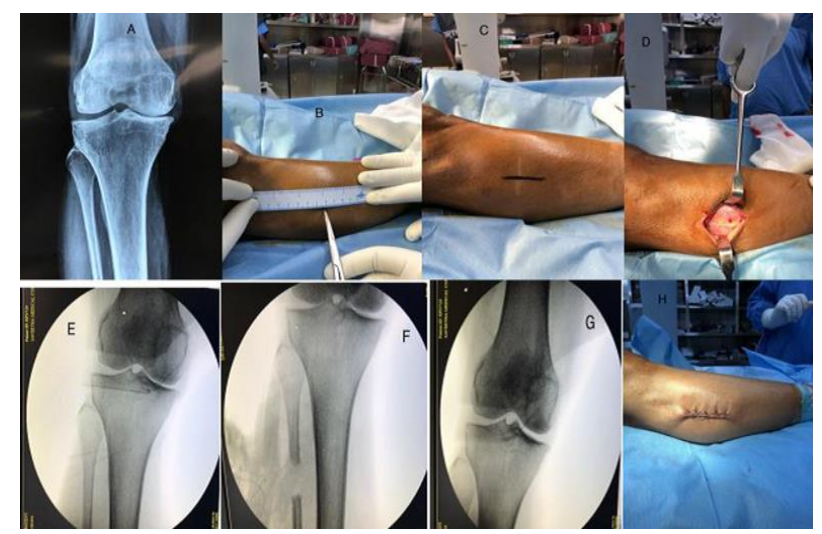

Fig (2) A:preoperative standing radiograph of knee showing decrease in medial joint space.B:marking a point 7-9cm from the head of fibula .C:Line of incision marked.D:Dissection carried out through perneus and soleus

muscles.E:C-ARM image prior to osteotomy.F:Fibular osteotomy done .G:Post osteotomy c-arm image showing an increase in medial joint space .H:Wound closure

\subsection{Statistical analyses}

The data collected was analyzed using IBM SPSS Version 22.0. Armonk, NY:IBM Corp. Chi square test was used in the comparison of categorical variables. A $\mathrm{P}$ value of less than 0.05 was considered to be statistically significant .

\section{Results}

This study shows the early results of proximal fibular osteotomy in medial compartment knee osteoarthritis , The knee function was assessed clinically, functionally and radiologically, three, six, nine, an twelve months postoperatively by Radiographic evaluation and Clinical assessment

Table (1) Patients' demographic characteristics.

\begin{tabular}{|c|c|c|c|}
\hline \multicolumn{4}{|l|}{-All patients $(n=20)$} \\
\hline & Mean \pm SD & Minimum & Maximum \\
\hline Age (years) & $56.60 \pm 6.30$ & 44.00 & 66.00 \\
\hline BMI & $28 \pm 4.38$ & 24 & 32 \\
\hline Gender & & $\begin{array}{l}40 \%(8) \\
60 \%(12)\end{array}$ & \\
\hline \multicolumn{4}{|c|}{ Data is expressed as mean and standard deviation, Minimum, Maximum. } \\
\hline \multirow{3}{*}{ Osteoarthritis grade } & & II & $20 \%(4)$ \\
\hline & & III & $70 \%(14)$ \\
\hline & & IV & $10 \%(2)$ \\
\hline \multirow{2}{*}{ Side } & & Right & $75 \%(15)$ \\
\hline & & Left & $25 \%(5)$ \\
\hline Data is expressed percentage a & ency. & & \\
\hline
\end{tabular}


Table (2) Medical history of osteoarthritis in the studied patients.

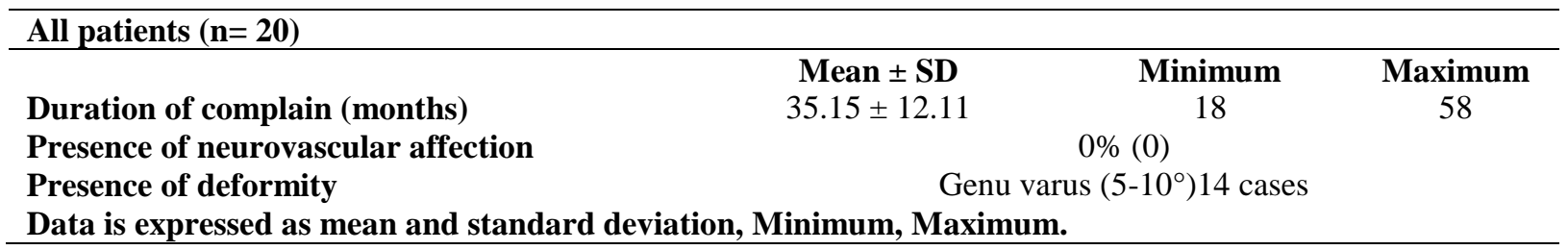

\section{Clinically \&functionally}

The knee function was assessed by Visual Analogue Score(VAS)and knee society score, the VAS which is a self report measure by patient himself which ranged preoperatively from 6 to 9 with mean was $7.35 \pm 0.745$ points ,after 3 months postoperatively ranged from 2 to 6 with mean $4.65 \pm 1.3$ and after 6 months from 0 to 5 with mean $3.15+-1.22$, and after 12 months postoperatively ranged from range from 0 to 3 points with mean was $1.4 \pm 0.995$ Table (.3)

Table (3) preoperative and follow-up values of VAS score.

\begin{tabular}{lccc}
\hline VAS score & Mean \pm SD & Minimum & All patients (n= 20) \\
\hline $\begin{array}{l}\text { Pre-operative } \\
\text { 3 months } \\
\text { postoperative } \\
\text { 6 months } \\
\text { postoperative }\end{array}$ & $7.35 \pm 0.745$ & 6 & Maximum \\
$\begin{array}{l}\text { 9 months } \\
\text { postoperative }\end{array}$ & $3.65 \pm 1.309$ & 2 & 6 \\
$\begin{array}{l}\text { 12 months } \\
\text { postoperative } \\
\text { Data is expressed as mean and standard deviation, Minimum, Maximum . }\end{array}$ \\
\hline
\end{tabular}

Clinical knee score: clinically ranged from 25 to 60 preoperative with mean 46 and increased 3 months postoperatively from 43 to 70 with mean 54 .

Function score: ranged from 20 to 60 preoperatively with median 35 and increased 3 months postoperatively from 30 to 70 with mean 50 .
Knee society score: is the sum of clinical knee score and function score which in totally show improvement from preoperative 28 to 70 with mean $46.80 \pm 12.668$ points and 3 months postoperatively was range from 36 to 79 points with mean $54.35 \pm$ 12.629 points after 12 months postoperative was range from range from 54 to 94 points with mean $82.05 \pm$ 10.73 points Table (4).

Table (4) preoperative and postoperative follow-up values of Knee Society score.

\begin{tabular}{llll}
\hline KSS score & \multicolumn{3}{l}{ All patients $(\mathbf{n}=\mathbf{2 0})$} \\
\hline & Mean $\mathbf{n}$ SD & Minimum & Maximum \\
\cline { 2 - 4 } Pre-operative & $46.80 \pm 12.668$ & 28 & 70 \\
3 months & $54.35 \pm 12.629$ & 36 & 79 \\
6 months & $63.45 \pm 13.736$ & 42 & 87 \\
9 months & $73.75 \pm 13.699$ & 50 & 93 \\
12 months & $82.05 \pm 10.738$ & 54 & 94 \\
Data is expressed as mean and standard deviation, Minimum, Maximum \\
\hline
\end{tabular}

\section{Radiographically}

Regarding medial and lateral knee joint space, the medial joint space was determined by a vertical line (A) between two horizontal lines (C and D) that were drawn from the lowest point of the medial condyle of the femur and medial plateau of the tibia, respectively. The lateral joint space was determined by a vertical line $(\mathrm{B})$ between two horizontal lines ( $\mathrm{E}$ and $\mathrm{F}$ ) that were drawn from the lowest point of the lateral condyle of the femur and lateral plateau of the tibia, respectively (figure1). In this study, radiographs of the weight-bearing lower extremity showed an average increase in the medial knee joint space postoperatively compared with preoperatively Table (5). And average decrease in the lateral knee joint space postoperatively compared with preoperatively Table (6). 
Table (5) preoperative and postoperative follow-up values of medial knee joint space.

\begin{tabular}{lccc}
\hline Medial joint space measured by mm. & & & All patients (n= 20) \\
\hline & Mean $\mathbf{\text { SD }}$ & Minimum & Maximum \\
\cline { 2 - 4 } Preoperative & $2.3+-0.8$ & 5 & 2.1 \\
3 months & $3.8+-.5$ & 2.3 & 4.3 \\
6 months & $4.2+-.6$ & 3.6 & 4.8 \\
9 months & $6.5+-.7$ & 5.8 & 7.2 \\
12 months & $7.2+-.1$ & 6.1 & 7.6 \\
Data is expressed as mean and standard deviation, Minimum, Maximum in $\mathbf{~ m m}$ & \\
\hline
\end{tabular}

Table (6) Comparison of preoperative and postoperative follow-up values of lateral knee joint space.

\begin{tabular}{lccc}
\hline Lateral kneejoint space & \multicolumn{3}{c}{ All patients $(\mathbf{n}=\mathbf{2 0})$} \\
\hline & Mean $\mathbf{\text { SD }}$ & Minimum & Maximum \\
\cline { 2 - 4 } Preoperative & $7.5 \pm 0.958$ & 7.1 & 7.8 \\
3 months & $7.2 \pm 0.842$ & 6.9 & 7.5 \\
6 months & $6.3 \pm 1.035$ & 5.8 & 6.7 \\
9 months & $5.7 \pm 1.185$ & 5.1 & 6.2 \\
12 months & $5.26 \pm 1.386$ & 4.9 & 5.4 \\
Data is expressed as mean and standard deviation,minimum,maximum in $\mathbf{~ m m . ~}$
\end{tabular}

\section{Complication}

Four patients had numbness of lateral aspect of foot,and three patients had extensor hallicis longus weakness.one of three patients had improved of big toe extension immediately during $2^{\text {nd }}$ month of follow up while all the patients showed improvement during the follow up .

\section{Discussion}

Knee osteoarthritis is one of the most widely recognized joint problems, and it causes serious torment and stability. Proximal fibular osteotomy (PFO) has developed as another medical procedure to assuage torment and improve joint capacity in patients with knee osteoarthritis [1].

Contrasted and absolute knee arthroplasty or high tibial osteotomy, PFO is a straightforward, sheltered, quick and reasonable medical procedure that doesn't need inclusion of extra embeds. Thusly, PFO is a reasonable careful choice in most non-industrial nations that need budgetary and clinical assets [2].

This medical procedure can possibly turn into an elective therapy strategy for osteoarthritis of the average compartment of the knee, particularly for patients who can't go through TKA due to clinical comorbidities [7].

The instrument of the viability of PFO is indistinct. One potential clarification of why PFO mitigates torment and improves the joint space is that it eliminates the fibula uphold that may cause genu varus. The fibula underpins one-6th of the body weight; along these lines, PFO may rebalance or reallocate the heap on the parallel and average tibia level after medical procedure [8].

Another conceivable instrument is nonuniform settlement as proposed by Yang et al. They expressed that the horizontal help gave to the osteoporotic tibia by the fibula delicate tissue complex may prompt

nonuniform settlement and degeneration of the level respectively, which may make the heap from the typical circulation move farther medially to the average level, subsequently prompting knee varus and exasperating the movement of average compartment osteoarthritis of the knee joint [2].

Regardless of whether the methodology doesn't give great outcomes in any circumstance then the field for playing out a Total knee arthroplasty at a later stage isn't changed in any way [9]. There is an overall lack of distributed writing on the function of proximal fibular osteotomy in average compartment osteoarthritis of the knee [10].

An investigation on 110 patients followed up for a very long time found that proximal fibular osteotomy can fundamentally improve both the radiographic appearance and capacity of the affected knee joint and furthermore accomplish long haul help with discomfort [2].

In this study,KSS scores indicated a huge increment ( $\mathrm{p}<0.001$ ) from 46.80 preoperatively up to 54.35 , $63.45,73.75$, and 82.05 at the recently booked subsequent visits separately.

In another examination, the mean knee and capacity subscores of the American Knee Society score were 44.41 and 41.24, individually. Postoperatively, they fundamentally improved to 69.02 and 67.63, individually [3].

Wang et al. From January 2015 to May 2015, 47 patients who went through proximal fibular osteotomy for average compartment osteoarthritis. One of 47 patients who went through PFO was lost to development, leaving 46 patients who were followed for at least a year. Average relief from discomfort was 
seen in all patients after PFO. The mean visual simple scale scores essentially diminished from 8.02 preoperatively to 2.74 postoperatively. Preoperatively, the mean knee and capacity subscores of the American Knee Society score were 44.41 and 41.24 , individually. Postoperatively, they altogether improved to 69.02 and 67.63, individually [3].

An as of late distributed Egyptian investigation detailed that Knee score which went from 24 to 55 preoperatively with middle 43.5 and expanded two months postoperatively from 40 to 67 with middle 55 . Also, Function score which went from 20 to 60 preoperatively with middle 35 and expanded two months postoperatively from 30 to 70 with middle 50 . Thus, Knee society score which is the amount of knee score and capacity score which in absolutely show improvement from preoperative middle 83 to 105 postoperatively [11].

It was accounted for that average help with discomfort was seen in all patients after PFO. The mean visual simple scale scores altogether diminished from 8.02 preoperatively to 2.74 postoperatively [3].

Concentrate by Zhang et al. in 2015 gave a gathering of 38 patients early OA and analyzed their preoperative and postoperative scores utilizing VAS Score. As indicated by their discoveries the VAS Score improved from 7 pre-operatively to 2.58 in the twentieth week post-usable development (Zhang, 2015). Another ongoing investigation additionally announced similar pattern with the VAS score improving from 7.89 preoperatively to 2.74 in their half year postoperative development [12].

A similar investigation of high tibial osteotomy(HTO) versus proximal fibula osteotomy, Proximal fibular osteotomy (PFO) is an elective treatment to high tibial osteotomy (HTO),It is a surgery for average compartment knee osteoarthritis (KOA). Contrasted with HTO, PFO has a few points of interest .First, the careful method is straightforward and effectively per-framed. Second, it is less obtrusive with a short cut, requires restricted tissue dismemberment and no inner obsession is embedded. The postoperative recuperation period is likewise more limited than with HTO. What's more, the intricacies related with HTO can be a significant issue that adds to a helpless forecast. Interestingly, PFO is related with hardly any confusions . Like HTO, PFO can calm the side effects of KOA with realignment of the lower furthest point. Be that as it may, while rectifying the arrangement is the significant goal of HTO [13].

The constraints of our examination were an overall number of patients, moderately short subsequent period and nonattendance of a benchmark group. A more extended time of follow up is important to assess whether the gainful impacts of PFO are continued throughout some undefined time frame.

\section{Conclusion}

Proximal fibular osteotomy (PFO) is a surgery for average compartment knee osteoarthritis (KOA).
Contrasted with HTO, PFO has a few focal points. To begin with, the careful strategy is straightforward and handily performed. Second, it is less intrusive with an exceptionally short cut, requires restricted tissue dismemberment and no inward obsession is embedded. The postoperative recuperation period is likewise more limited than with HTO. Furthermore, the confusions related with HTO can be a significant issue that adds to a helpless taking everything into account, we suggest proximal fibular osteotomy for average compartment knee O.A. as it is insignificantly obtrusive, straightforward, sheltered, quick and moderate technique particularly in youthful patient.

\section{References}

[1] A.Amendola, D.E.Bonasia. Results of high tibial osteotomy: review of the literature. Int Orthop, Vol.34 (2),PP. 155-160,2010.

[2] A.Amendola, D.E.Bonasia, Results of high tibial osteotomy: review of the literature. Int Orthop,Vol.34(2),PP.155-160,2010.

[3] T.Baldini, J. Roberts,J.Hao, K.Hunt,M.Dayton, Medial Compartment Decompression by Proximal Fibular Osteotomy: A Biomechanical Cadaver Study. Orthopedics,Vol.41(4),PP. 496-501, 2018.

[4] T.Dong, W.Chen, F.Zhang, B.Yin, Y.Tian, Y.Zhang, Radiographic measures of settlement phenomenon in patients with medial compartment knee osteoarthritis.Clin

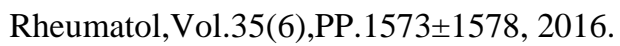

[5] A.Elsebaiy, H.Safwat, and A. A. R. Ashiry, Early Results of Fibular Osteotomy for Treatment of Medial Compartment Osteoarthritis. The Egyptian J., of Hospital Medicine,Vol.76(7),PP.45884590,2019.

[6] S.Fooladi, A. Vaish, Y. Nigam, and R.Vaishya, Joint preservation in a middle-aged man with knee osteoarthritis, using novel techniques. Apollo Medicine,Vol.16(2),PP.124-126, 2019.

[7] D.Ghinelli, A.Parma, M.Baldassarri, A.Olivieri, M.Mosca, G.Pagliazzi, High tibial osteotomy for the treatment of medial osteoarthritis of the knee with new iBalance system: 2 years of follow-up. Eur J Orthop Surg Traumatol,Vol.26(5),PP.113,2016

[8] R. K. Misra, and A. V. Batra, Clinical and Functional Outcomes of Proximal Fibular Osteotomy on Varus Deformity and Medial Compartment Knee Osteoarthritis. J., of Arthritis,Vol.8(281),PP.225-229,2019.

[9] A.Amendola, D.E.Bonasia. Results of high tibial osteotomy: review of the literature. Int Orthop,Vol.34(2),PP.155 \pm 160 ,

2010.https://doi.org/10.1007/ s00264 -009-0889-8 PMID: 19838706

[10] L. Prakash, Proximal Fibular Osteotomy for Medial compartment arthritis of the knee joint. Elsebaiy, A. ,Vol.22,PP.96-101,2018. 
[11] H.Safwat, and A. A. R. Ashiry, Early Results of Fibular Osteotomy for Treatment of Medial Compartment Osteoarthritis. The Egyptian J., of Hospital Medicine, 76(7), 4588-4590,2019.

[12] Y.Subash, and G. K. Naidu, The role of proximal fibular osteotomy in the management of medial compartment osteoarthritis of the knee. International J., of Orthopaedics,Vol4(3),PP.369372,2018 .

[13] X.Wang, L.Wei, Z. Lv, B.Zhao, Z.Duan, W.Wu, Proximal fibular osteotomy: a new surgery for pain relief and improvement of joint function in patients with knee osteoarthritis. J., of International Medical Research, Vol.45(1),PP. 282-289, 2017.

[14] Z. Y. Yang, W.Chen, C. X.Li, J.Wang, Z. Y.Hou, Medial compartment decompression by fibular osteotomy to treat medial compartment knee ---osteoarthritis: a pilot study. Orthopedics, Vol. 38(12),PP.1110- 1114,2015.

[15] Y. Z. Zhang, Innovations in orthopedics -and traumatology in China.Chinese medical J., Vol.128(21),PP. 2841-2843,2015. 